\title{
Co/N co-doped graphene-like nanocarbon for highly efficient oxygen reduction electrocatalyst
}

\author{
Lei Liu ${ }^{1}$, Jian Zhang ${ }^{1,2}$, Wujun $\mathrm{Ma}^{3}$ and Yunhui Huang ${ }^{1 *}$
}

\begin{abstract}
The development of efficient and inexpensive graphene-based electrocatalysts is of great significance to promote the commercial application of fuel cell and metal-air batteries. In this paper, a new type of Co and $\mathrm{N}$ co-doped graphene-like nanocarbon (Co/N-GLC) material was prepared by nano-silicon protection and high temperature pyrolysis. The obtained Co/N-GLC catalyst not only has a similar morphology of graphene, but also possesses a high specific surface area $\left(809 \mathrm{~m}^{2} \mathrm{~g}^{-1}\right)$ with hierarchical porous structure (micropores/mesopores), and relative high active dopants content. These properties endow it with a good oxygen reduction activity in alkaline media, which can be comparable to commercial $\mathrm{Pt} / \mathrm{C}$ catalyst. Moreover, the assembled zinc-air batteries using Co/N-GLC catalyst as the air electrode display a better discharge performance and higher stability compared to that of $\mathrm{Pt} / \mathrm{C}$ electrode. This work demonstrates that the prepared graphene-like carbon catalyst has a good prospect, which can replace noble metal catalyst at the cathode in metalair batteries.
\end{abstract}

Keywords: graphene-like nanocarbon, oxygen reduction reaction, electrocatalyst, zinc-air battery

\section{INTRODUCTION}

The development of stable and highly efficient electrocatalyst for oxygen reduction reaction (ORR) in the cathode is extremely essential for metal-air batteries and fuel cells [1-4]. Nowadays, Pt-based noble metal nanomaterials are considered as the most promising catalyst for ORR [5]. However, they usually suffer from high price, rare resource, susceptibility to fuel (e.g., methanol and $\mathrm{CO}$ ), and poor durability [6,7], which remain major challenges for industry application. Thus, more and more researchers are devoted to exploring efficient and durable non-noble metal ORR catalysts with low-cost earth- abundant materials.

Graphene, a unique flat monolayer of carbon atoms, has attracted a great deal of interests for ORR due to its novel two-dimensional (2D) structure, ultra-high specific surface area (SSA), high electrical conductivity and flexibility, and good chemical stability [7-9]. After heteroatom doping and compositing with transition metal, this hybrid graphene based nanomaterials have gained a better ORR activity with higher stability in alkaline solution $[1,10,11]$. However, so far, the synthetic route of graphene is very complicated and time-consuming [8], which hinders its large-scale application. In addition, owing to the inherent strong van der Waals force, stacking and agglomeration are easy to occur on graphene nanomaterials, resulting in the decrease of SSA and porosity $[12,13]$. These drawbacks limit the number of active sites and hamper the diffusion of reaction ions, thereby reducing the overall electrocatalytic performance for ORR. Fortunately, recent studies demonstrate that the graphenelike nanomaterials can efficiently inhibit the inherent $\pi-\pi$ restacking, resulting in high SSA and porosity, and simultaneously keep the similar properties with graphene (e.g., high electronic conductivity, superior structural stability) $[1,6,12,13]$. Thus, these graphene-like nanomaterials possess a good performance in the energy conversation and storage field, which can be comparable or even superior to that of pristine graphene [14].

As well-known that specific heteroatom-doping and metal coordination at defective edges are the most favorable active sites [15-18]. Based on the above research, we develop a new type of $\mathrm{Co}$ and $\mathrm{N}$ co-doped graphenelike nanocarbon (Co/N-GLC) material via a simple and cost-efficient strategy of nano-silicon protection and high temperature pyrolysis. The as-prepared $\mathrm{Co} / \mathrm{N}-\mathrm{GLC}$ catalyst not only has thin carbon-layer nanostructure and

${ }^{1}$ School of Materials Science and Engineering, Huazhong University of Science and Technology, Wuhan 430074, China

${ }^{2}$ School of Materials Science and Engineering, Nanyang Technological University, Singapore 639798, Singapore

${ }^{3}$ School of Chemistry, Biology and Materials Engineering, Suzhou University of Science and Technology, Suzhou 215009, China

* Corresponding author (email: huangyh@hust.edu.cn) 
high SSA with excellent micro/mesoporisity, but also contains high levels of active heteroatom dopants. The electrochemical measurements show that the graphenelike catalyst has a comparable ORR activity with commercial $\mathrm{Pt} / \mathrm{C}$ in alkaline media. What's more, when used as the air electrode catalyst for zinc-air batteries, it exhibits a favorable discharge performance and stability compared to $\mathrm{Pt} / \mathrm{C}$ electrode.

\section{EXPERIMENTAL SECTION}

\section{Preparation of Co/N-GLC catalyst}

For the first step, $2 \mathrm{~g}$ cobaltous acetate and $3.2 \mathrm{~g}$ orthophenanthroline were dissolved in 500 and $50 \mathrm{~mL}$ ethanol separately to form homogeneous solution A and B by vigorously stirring. Then, $5 \mathrm{~g}$ magnesium hydroxide powder was added during the process of the solution $\mathrm{B}$ dropped slowly into the solution A. After $1 \mathrm{~h}$ at $80^{\circ} \mathrm{C}$, the mixed solution was constantly stirred for $20 \mathrm{~h}$ at room temperature. The obtained product was heated at $400^{\circ} \mathrm{C}$ for $30 \mathrm{~min}$ under nitrogen atmosphere. Then, the sample was taken out and mixed with $2 \mathrm{~mL}$ formic acid and $2 \mathrm{~mL}$ tetraethylorthosilicate. Then, the resultant suffered a further heat treatment at $900^{\circ} \mathrm{C}$ for $1 \mathrm{~h}$ under nitrogen flow. At last, the black powder was washed by the mixed solution of dilute sulphuric acid and hydrofluoric acid to remove nano-silicon and residue. After thoroughly washing and drying, the Co/N-GLC catalyst was collected with a yield of $0.2147 \mathrm{~g}$ (Fig. S1). To investigate the effects of Co source and silicon protection on the ORR activity of the catalyst, the contrast samples without Co species and silicon protection (named as $\mathrm{N}-\mathrm{C}$ and $\mathrm{Co} / \mathrm{N}-\mathrm{C}$, respectively) were also prepared through the same synthetic procedure except for not using cobaltous acetate and tetraethylorthosilicate, separately.

\section{Characterization}

Field-emission scanning electron microscope (FE-SEM, Sirion 200), and high-resolution transmission electron microscope (HR-TEM, Tecnai G2F30) were employed to investigate the morphology and structure of the samples. The crystalline structure of the materials was thoroughly characterized with powder X-ray diffractometer (XRD) between $10^{\circ}$ and $80^{\circ}$ with $\mathrm{Cu} \mathrm{Ka}$ radiation. The $\mathrm{N}_{2}$ adsorption/desorption curves and the pore size distribution (PSD) were obtained from Micromeritics analyzer $(3 \mathrm{H}-$ 2000PM) measurements, whereas the SSA data were calculated on the basis of the Brunauer-Emmett-Teller (BET) model. The chemical composition was analyzed by X-ray photoelectron spectrometer (XPS, AXIS-ULTRA
DLD-600W) conducted with monochromatic $\mathrm{Al} \mathrm{Ka}$ radiation $(1,486.71 \mathrm{eV})$. Raman spectroscopy was recorded by a Renishaw instrument via Ar ion laser at an excitation line of $514.5 \mathrm{~nm}$.

\section{Electrocatalytic performance of $\mathrm{Co} / \mathrm{N}$-GLC catalyst}

All electrochemical measurements were performed at room temperature with an electrochemical workstation (CHI660E) in a conventional three-electrode system. Firstly, the catalyst ink was prepared by ultrasonic mixing of $5.0 \mathrm{mg}$ catalyst into a solution containing ethanol $(0.475 \mathrm{~mL})$ and Nafion electrolyte $(25 \mu \mathrm{L}, 5 \mathrm{wt} \%, \mathrm{Du}-$ Pont) to a homogeneous suspension. After ultrasonication for $1 \mathrm{~h}$, an $8 \mu \mathrm{L}$ aliquot of ink was evenly drop casted on a clean glass carbon disk ( $5 \mathrm{~mm}$ diameter) surface and dried in air condition as a working electrode (mass loading of $0.16 \mathrm{mg} \mathrm{cm}^{-2}$ ). Pt foil and saturated calomel electrode served as the counter and reference electrodes, respectively. As a benchmark, the state-of-the-art commercial Pt/C (Johnson Matthey, 20\%) was coated on the working electrode by the same method. The ORR activity of all samples was measured through cyclic voltammetry (CV) in $\mathrm{O}_{2}$-saturated $0.1 \mathrm{~mol} \mathrm{~L}^{-1} \mathrm{KOH}$ electrolyte. The electrode was scanned at a rate of $20 \mathrm{mV} \mathrm{s}^{-1}$. Linear sweep voltammetry (LSV) for ORR experiments were carried out using a rotating disk electrode (RDE) technique with a scan rate of $10 \mathrm{mV} \mathrm{s}^{-1}$ in an $\mathrm{O}_{2}$-saturated $0.1 \mathrm{~mol} \mathrm{~L}^{-1}$ $\mathrm{KOH}$ aqueous solution.

A laboratory-made $\mathrm{Zn}$-air battery (ZAB) was also fabricated, where the $\mathrm{Co} / \mathrm{N}-\mathrm{GLC}$ or commercial $\mathrm{Pt} / \mathrm{C}$ catalyst was sprayed onto hydrophobic carbon paper (Toray TGP-H-090), a polished $\mathrm{Zn}$ plate, and $6 \mathrm{~mol} \mathrm{~L}^{-1} \mathrm{KOH}$ were employed as the air cathode, anode, and aqueous electrolyte, respectively. The catalyst loading was calculated to be $1 \mathrm{mg} \mathrm{cm}^{-2}$ and the amount of electrolyte was $5 \sim 6 \mathrm{~mL}$.

\section{RESULTS AND DISCUSSION}

Morphology of Co/N-GLC catalyst was studied by FESEM and HR-TEM. As revealed in Fig. 1a, a large number of macroporous structures irregularly distribute on the Co/N-GLC. Additionally, some mesopores can also be

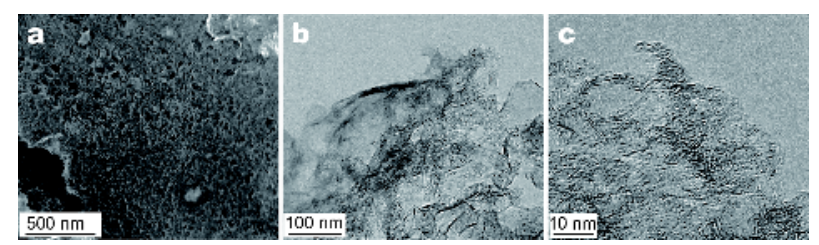

Figure 1 FE-SEM (a) and HR-TEM (b, c) images of Co/N-GLC. 
observed in Fig. 1b. This is primarily attributed to the addition of $\mathrm{Mg}(\mathrm{OH})_{2}$, which plays a pore-former agent during the pyrolysis process [6]. Interestingly, some subtransparent carbon nanosheets with wrinkles exist in the Co/N-GLC. The HR-TEM image of Fig. 1c suggests that these carbon nanosheets are mainly composed of few-layer carbon layers, similar as the morphology of graphene $[7-9,19]$. This may be because that the nanosilicon protective layer effectively prevents the aggregation and stacking of the carbon layers during the heat treatment at high temperature, and the catalytic effect of the metal cobalt also promotes the formation of the carbon nano-flake simultaneously [20]. Their lattice fringes are shorter and highly disordered, especially on the edge, indicating that amounts of defective sites exist in the $\mathrm{Co} / \mathrm{N}$-GLC, which are beneficial for increasing the number of electrocatalytically active sites for ORR [11,2123]. Noteworthy, no metal particles are observed in the $\mathrm{Co} / \mathrm{N}-\mathrm{GLC}$, suggesting that most of metal cobalt or compounds are removed by harsh acid-leaching, and the residual cobalt atoms atomically bind with carbon-nitrogen framework to form the active sites $[19,24]$.

As shown in Fig. 2a, the crystal structure of the asprepared $\mathrm{Co} / \mathrm{N}-\mathrm{GLC}$ catalyst was further characterized with XRD. The two obvious peaks located at about $26.8^{\circ}$ and $43.0^{\circ}$ in the XRD spectrum are assigned to the (002) and (101) planes (JCPDS No. 75-1621) of graphite carbon $[6,25]$. The broader and smooth $(002)$ peak indicates the crystal structure of $\mathrm{Co} / \mathrm{N}-\mathrm{GLC}$ is largely disordered with a low degree of graphitization, consistent with the HRTEM results [23]. Furthermore, no sharp peak can be found in the XRD pattern, showing that neither metal Co nor cobalt oxide nanoparticle exists in the sample. In Fig. $2 \mathrm{~b}$, Raman spectrum of the $\mathrm{Co} / \mathrm{N}-\mathrm{GLC}$ is characterized by three main peaks D, G and 2D. Generally, the D- and Gband are associated with the defects or disorder in graphite carbon and the vibration of $\mathrm{sp}^{2}$-bonded carbon atoms in the hexagonal graphitic lattice, respectively $[13,26]$. The intensity ratio of the two bands $\left(I_{\mathrm{D}} / I_{\mathrm{G}}\right)$ is usually used to estimate the density of defects in carbon materials $[11,21]$. The strong D-band along with high intensity ratio of $I_{\mathrm{D}} / I_{\mathrm{G}}(1.22)$ is attributed to the large number of structural defects within the Co/N-GLC. Researchers have claimed that the defects in carbon based catalysts could create more active sites to increase the catalytic active density [27-30]. In addition, Raman spectrum shows a well-defined 2D-band (the typical characteristic band of graphene), further proving the graphene-like structure of the Co/N-GLC.

As shown in Fig. 2c, the porous structure characteristic and BET surface area of the Co/N-GLC were measured by $\mathrm{N}_{2}$ adsorption-desorption isotherms. The curve shows a mixture of type I and IV isotherm, indicative of the presence of micro- and mesosize pore in the $\mathrm{Co} / \mathrm{N}-\mathrm{GLC}$ $[12,13,30,31]$, which matches well with the pore size distribution of the Co/N-GLC in Fig. 2d. BET surface area and total pore volume of the Co/N-GLC are $809 \mathrm{~m}^{2} \mathrm{~g}^{-1}$ and $1.30 \mathrm{~cm}^{3} \mathrm{~g}^{-1}$, respectively. As previous reports $[22,29,32]$, the high surface area creates more active sites for ORR, and the hierarchical porous structure promotes the ORR, leading to the improvement of the overall ORR activity.

XPS measurement confirms the surface composition and chemical status of the Co/N-GLC (Fig. 2e). The survey XPS spectrum shows that the Co/N-GLC catalyst predominantly consists of $\mathrm{C}, \mathrm{N}, \mathrm{O}$, as well as a small amount of Co atoms, corresponding to 83.89, 2.53, 12.97 and 0.61 at.\%, respectively. For accurate determination of Co content, inductively coupled plasma mass spectrometry (ICP-MS) was performed. The result shows that the amount of Co atoms in the Co/N-GLC catalyst is $1.63 \%$. It should be noted that neither the HR-TEM nor the XRD measurements indicate the existence of metal cobalt or compounds in the $\mathrm{Co} / \mathrm{N}-\mathrm{GLC}$, implying that the cobalt atoms are highly dispersed in the inner part of $\mathrm{Co} /$ $\mathrm{N}-$ GLC, and covalently combined with carbon-nitrogen bonds to form catalytic activity sites [6,14]. Additionally, no peak of Mg and Si in XPS spectrum is observed, indicating that these two elements are thoroughly removed by acid washing. It is well-known that the $\mathrm{N}$ doped sites play the significant roles in determining the ORR activity. Generally, pyridinic-N (PN) can improve the onset potential and boost the wettability of the carbon-based electrocatalysts; while graphitic-N (GN) can increase the limiting current density. However, other types of $\mathrm{N}$ dopants have little effect on the electrocatalytic activity $[13,33]$. As shown in the fitted result of N 1s (Fig. 2f), Co/ N-GLC contains a high ratio of PN (51.5\%) and GN (35.1\%) dopants, implying that it has good ORR activity.

To assess the ORR activity of Co/N-GLC, LSV and CV measurements were performed in Fig. 3. For comparison, the commercial Pt/C (20 wt.\%) was also tested under the same conditions. As displayed in Fig. 3a, Co/N-GLC shows the highest onset $\left(E_{0}=0.966 \mathrm{~V}\right)$ and half-wave $\left(E_{1 / 2}=0.946 \mathrm{~V}\right)$ potentials compared to the contrast samples without silicon protection $(\mathrm{Co} / \mathrm{N}-\mathrm{C})$ and the absence of Co source $(\mathrm{N}-\mathrm{C})$, demonstrating that both of Co source and silicon protection play a key role in improving ORR activity for the Co/N-GLC catalyst. Remarkably, the $E_{0}$ and $E_{1 / 2}$ of Co/N-GLC are only 8 and $85 \mathrm{mV}$ negative 

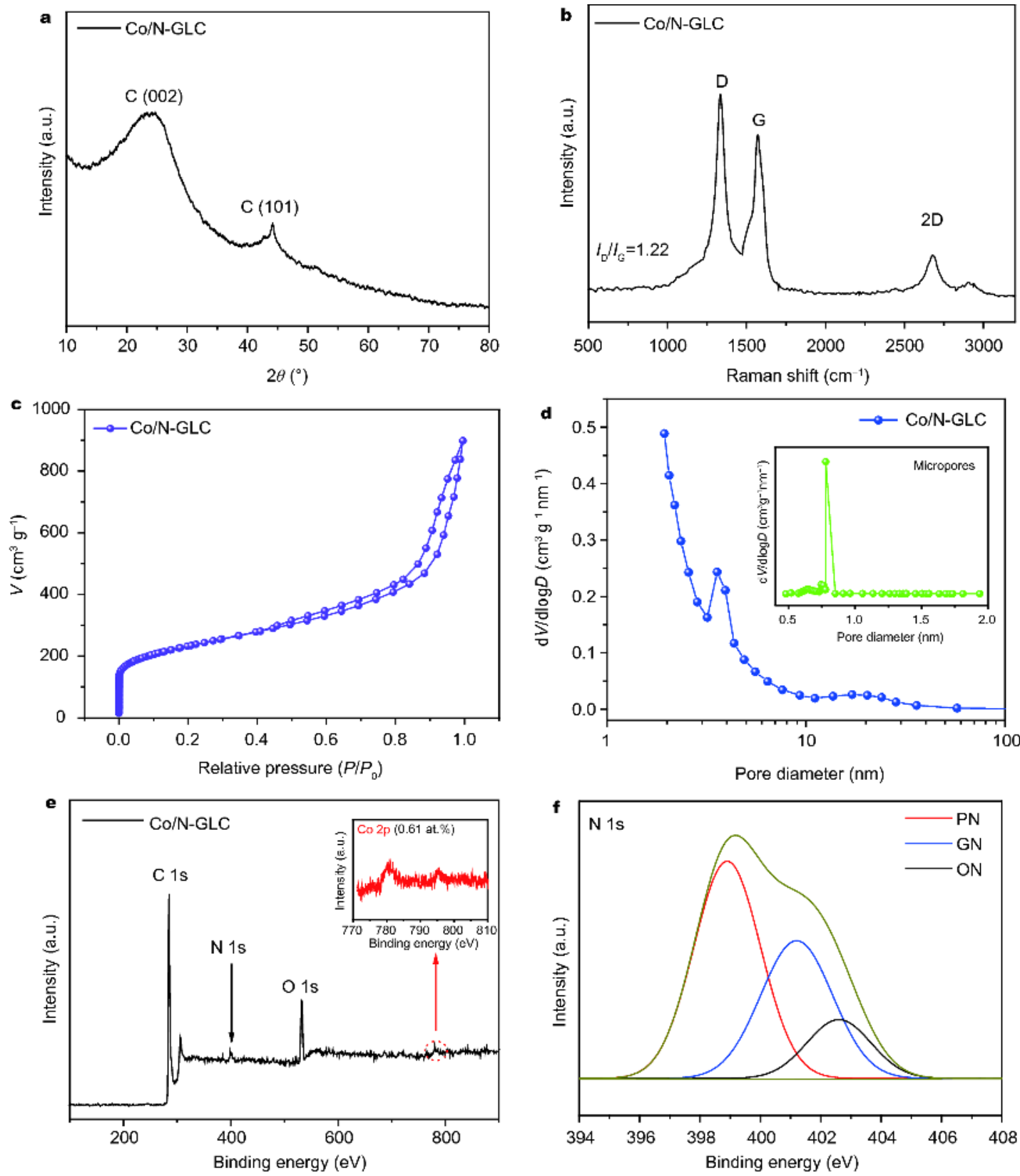

Figure 2 (a) XRD pattern, (b) Raman spectrum, (c) $\mathrm{N}_{2}$ adsorption-desorption isotherms, (d) pore size distributions (the inset is micropore distribution), (e) XPS spectra (the inset is Co $2 \mathrm{p}$ spectrum) and (f) the fitted results of N 1s of Co/N-GLC.

than those of $\mathrm{Pt} / \mathrm{C}$ catalyst $\left(E_{0}=0.974 \mathrm{~V}\right.$ and $E_{1 / 2}=$ $0.861 \mathrm{~V}$ ), respectively. CV curves (Fig. 3b) reveal that the ORR peak potential of $\mathrm{Co} / \mathrm{N}-\mathrm{GLC}$ is up to $0.826 \mathrm{~V}$, which is also slightly lower than that of $\mathrm{Pt} / \mathrm{C}$ catalyst $(0.839 \mathrm{~V})$, further demonstrating the good ORR activity of $\mathrm{Co} / \mathrm{N}$ GLC. In addition, the electrochemical active surface area (ECSA) of Co/N-GLC was also determined via the scanrate dependent $\mathrm{CV}$ measurements. As exhibited in Fig.
S2, Co/N-GLC, owing to the high surface area and enriched micro/mesopores (Fig. $2 \mathrm{c}$ and d), shows a high ECSA represented by the double layer capacitance $\left(6.9 \mathrm{mF} \mathrm{cm}{ }^{-2}\right)$, indicative of high density of active sites for ORR.

In Fig. $3 c$, the reaction kinetics of $\mathrm{Co} / \mathrm{N}-\mathrm{GLC}$ catalyst were evaluated via the different rotation speed within the range from 400 to $2,000 \mathrm{rpm}$. The five corresponding 

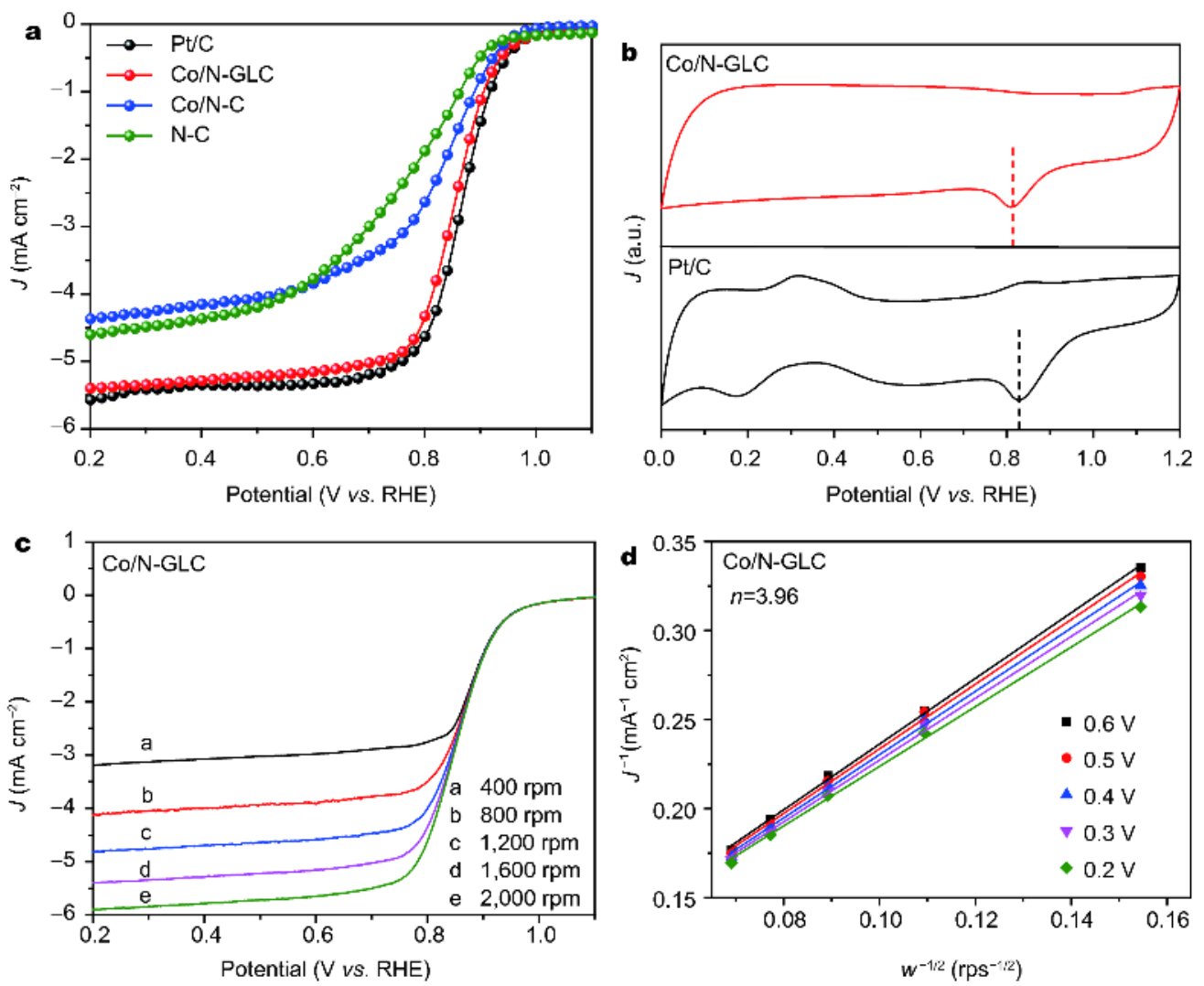

Figure 3 (a) LSV curves of the commercial Pt/C, Co/N-GLC, Co/N-C and N-C catalysts. (b) $\mathrm{CV}$ curves in $\mathrm{O}_{2}$ saturated $0.1 \mathrm{~mol} \mathrm{~L} \mathrm{~L}^{-1} \mathrm{KOH}$ for Co/NGLC and Pt/C; (c) LSV curves for Co/N-GLC at different rotation rates and (d) the corresponding K-L plots at different potentials.

Koutecky-Levich (K-L) plots clearly show a good parallel linear relationship from 0.2 to $0.6 \mathrm{~V}$ (Fig. 3d), consistent with the first-order reaction kinetics of ORR [24]. The electron transfer number $(n)$ of the catalyst during the ORR is commonly evaluated using the K-L equation $[6,13]$ :

$$
\begin{gathered}
\frac{1}{J}=\frac{1}{J_{\mathrm{L}}}+\frac{1}{J_{\mathrm{K}}}=\frac{1}{B \omega^{1 / 2}}+\frac{1}{J_{\mathrm{K}}}, \\
B=0.62 n F C_{0}\left(D_{0}\right)^{2 / 3} v^{-1 / 6},
\end{gathered}
$$

where $J, J_{\mathrm{K}}$, and $J_{\mathrm{L}}$ denote the measured, kinetic, and diffusion-limited current densities, respectively, $\omega$ is the electrode rotation rate, $F$ is the Faraday constant $\left(96,485 \mathrm{C} \mathrm{mol}^{-1}\right), C_{0}$ is the bulk concentration of $\mathrm{O}_{2}$ $\left(1.2 \times 10^{-3} \mathrm{~mol} \mathrm{~L}^{-1}\right), D_{0}$ is the diffusion coefficient of $\mathrm{O}_{2}$ $\left(1.9 \times 10^{-5} \mathrm{~cm}^{2} \mathrm{~s}^{-1}\right)$ and $v$ is the kinetic viscosity of the electrolyte $\left(1.0 \times 10^{-2} \mathrm{~cm}^{2} \mathrm{~s}^{-1}\right)$. Referring to the above equation, the calculated average $n$ value of Co/N-GLC is 3.98, which is very similar to the four-electron transfer of $\mathrm{Pt} / \mathrm{C}$, demonstrating that $\mathrm{Co} / \mathrm{N}$-GLC mainly proceeds in a four-electron transfer process with low content of byproducts $[7,31]$. Additionally, we also investigated the
ORR performance of $\mathrm{Co} / \mathrm{N}$-GLC catalyst in acidic media. As displayed in Fig. S3, the onset potential of Co/N-GLC is up to $0.825 \mathrm{~V}$, which is $110 \mathrm{mV}$ negative than that of $\mathrm{Pt} / \mathrm{C}$ catalyst. However, the long-term current-time chronoamperometric response shows that the stability of $\mathrm{Co} / \mathrm{N}-\mathrm{GLC}$ catalyst ( $93 \%$ within $10,000 \mathrm{~s}$ ) is higher than that of $\mathrm{Pt} / \mathrm{C}$ catalyst ( $84 \%$ within $10,000 \mathrm{~s}$ ) in acidic media.

To unveil the ORR activity of the Co/N-GLC catalyst, we carried out density functional theory calculations on a typical $\mathrm{Co}$ and $\mathrm{N}$ co-doped graphene $(\mathrm{Co} / \mathrm{N}-\mathrm{G})$ model [13] to explore the charge and spin distribution on the atoms. The detailed calculation process can be seen in the Supporting information. As shown in Fig. $4 \mathrm{a}$ and $\mathrm{b}$, the center of Co atom shows the highest positive charge and spin density, which are in favor of $\mathrm{O}_{2}$ adsorption and reduction according to recent reports [27,28]. Additionally, the carbon atoms around nitrogen also show comparatively higher charge and spin density, which can further promote the $\mathrm{O}_{2}$ adsorption capability and serve as catalytically active sites for ORR [29]. Moreover, we calculated the highest-occupied molecular orbital (HOMO) 

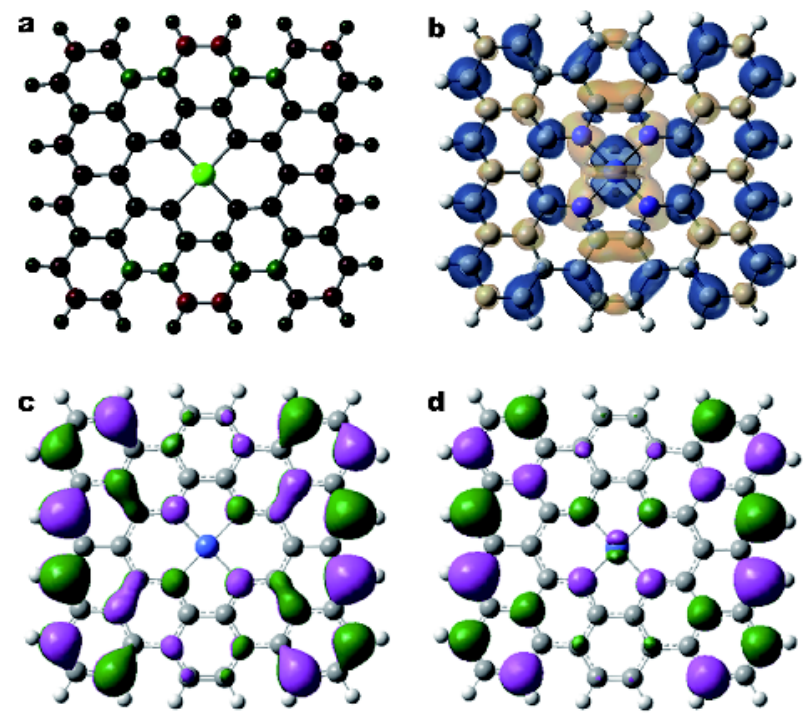

Figure 4 (a) The charge and (b) spin density, and (c) HOMO and (d) LUMO of Co/N-G model. The green and blue circles are labeled as Co and N atoms, respectively. The others are $\mathrm{C}$ atoms. All the carbon atoms on the edge of the graphene are terminated by hydrogen atoms.

to the lowest-unoccupied molecular orbital (LUMO) energy gap of $\mathrm{Co} / \mathrm{N}-\mathrm{G}$ models (Fig. $4 \mathrm{c}$ and $\mathrm{d}$ ). The HOMO and LUMO at the edges of the model are greatly polarized by the covalently bonded Co and $\mathrm{N}$ dopants. These polarized zones might also serve as the active sites for ORR $[34,35]$. A smaller HOMO-LUMO energy gap actually indicates a higher chemical reactivity, since it has a beneficial effect on adding electrons to a high-lying LUMO, to extract electrons from a low-lying HOMO, and facilitating the electron transfer and the overall reaction $[21,27,28]$. Thus, the low HOMO-LUMO energy gap of $\mathrm{Co} / \mathrm{N}-\mathrm{G}(0.44 \mathrm{eV})$ demonstrates the high catalytic performance.

Finally, to confirm the good electrocatalytic activity of $\mathrm{Co} / \mathrm{N}-\mathrm{GLC}$ in the actual application, the two electrodes of air cathode (carbon paper with $\mathrm{Co} / \mathrm{N}-\mathrm{GLC}$ loaded on) and $\mathrm{Zn}$ plate anode, were assembled into a laboratorymade ZAB. For comparison, the commercial $\mathrm{Pt} / \mathrm{C}$ catalyst is also investigated via the same procedure. Seen from Fig. 5a, the fabricated ZAB using Co/N-GLC electrode displays an identical open-circuit voltage (OCV) to that of $\mathrm{Pt} / \mathrm{C}$ electrode. A high voltage plateau of $1.32 \mathrm{~V}$ for the $\mathrm{Co} / \mathrm{N}-\mathrm{GLC}$ electrode appears in the galvanostatic discharge curves (Fig. 5b) at a current density of $5 \mathrm{~mA} \mathrm{~cm}^{-2}$. Notably, Co/N-GLC electrode keeps a highly stable voltage plateau after the long-term discharge, while the $\mathrm{Pt} / \mathrm{C}$ electrode displays a certain of voltage drop, indicating the good stability of Co/N-GLC catalyst. Fig. $5 \mathrm{c}$ shows the rate capability of battery catalyzed by $\mathrm{Co} / \mathrm{N}-\mathrm{GLC}$ electrode. With the increase of current densities from 2 to
$50 \mathrm{~mA} \mathrm{~cm}^{-2}$, all the discharge voltages maintain highly stable plateaus. Moreover, the discharge voltage can restore its original level when the current density returns to a low discharge current density, endowing the battery with an excellent rate capability. The remarkable electrocatalytic performance may primarily be contributed to the highly open hierarchical porous architectures in $\mathrm{Co} /$ $\mathrm{N}$-GLC, which are favorable for the reaction boundary of oxygen reduction by supplying a convenient electronic transmission path and facilitating mass transfer $[14,21,31]$. Remarkably, after long-term discharging at $20 \mathrm{~mA} \mathrm{~cm}^{-2}$, as displayed in Fig. 5d, the battery with $\mathrm{Co} /$ $\mathrm{N}$-GLC electrode almost has no voltage drop during multi cycles, further indicating the excellent stability of the ND-GLC catalyst for ORR. As expected, two seriesconnected ZABs assembled with $\mathrm{Co} / \mathrm{N}-\mathrm{GLC}$ air cathode could directly deliver enough energy to light up 10 parallel high-power red LEDs for a long time (Fig. 5e). The above results illustrate that the as-prepared $\mathrm{Co} / \mathrm{N}-\mathrm{GLC}$ material can be applied in practical applications as an alternative to $\mathrm{Pt} / \mathrm{C}$ catalyst.

\section{CONCLUSIONS}

In summary, we synthesized a new type of graphene-like (Co/N-GLC) catalyst via nano-silicon protection and high temperature pyrolysis. Benefiting from its similar morphology of graphene, high surface area and porosity, abundant edge defects, and high-content active dopants, the ORR activity of Co/N-GLC is close to that of commercial $\mathrm{Pt} / \mathrm{C}$ catalyst in alkaline media. When employed 

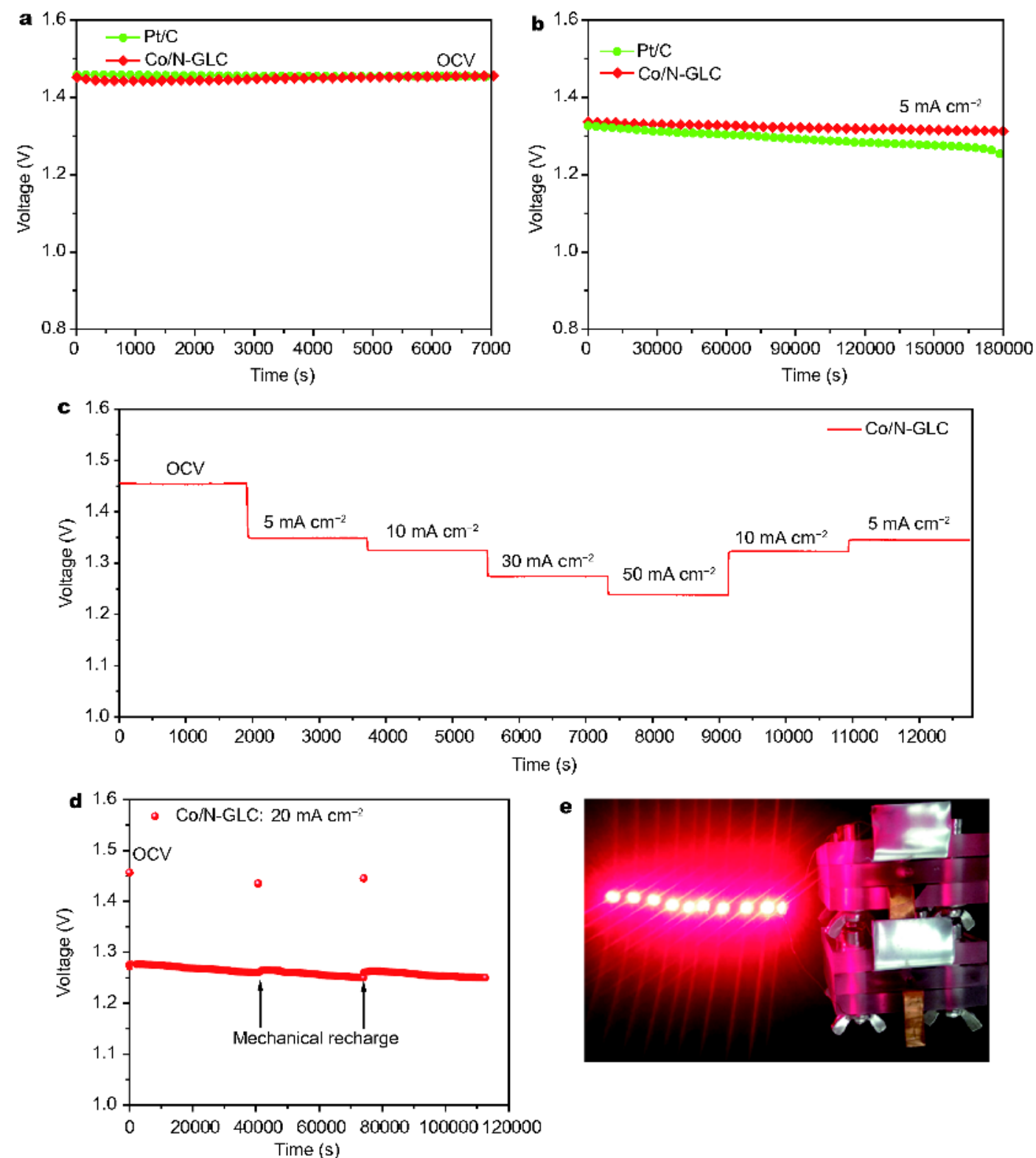

Figure 5 (a) Open-circuit voltage measurements and (b) discharge curves of ZABs based on Co/N-GLC and Pt/C at $5 \mathrm{~mA} \mathrm{~cm}{ }^{-2}$. (c) Discharge curve of ZAB based on Co/N-GLC at different current densities and (d) long time discharge curve at $20 \mathrm{~mA} \mathrm{~cm}{ }^{-2}$ by mechanical recharging. (e) Photograph of 10 parallel LED lamps driven by two ZABs based on Co/N-GLC.

as air cathode in ZAB, the $\mathrm{Co} / \mathrm{N}-\mathrm{GLC}$ catalyst also shows a comparable discharge performance in comparison with $\mathrm{Pt} / \mathrm{C}$ electrode, demonstrating that the prepared graphene-like carbon catalyst has the potential to replace noble metal catalyst in practical applications.

Received 25 May 2018; accepted 10 July 2018; published online 17 August 2018

1 Dai L, Xue Y, Qu L, et al. Metal-free catalysts for oxygen reduction reaction. Chem Rev, 2015, 115: 4823-4892

2 Borghei M, Lehtonen J, Liu L, et al. Advanced biomass-derived electrocatalysts for the oxygen reduction reaction. Adv Mater, 2018, 30: 1703691

3 Dou S, Tao L, Wang R, et al. Plasma-assisted synthesis and surface modification of electrode materials for renewable energy. Adv Mater, 2018, 30: 1705850-1705874

4 Wang S, Jiang SP. Prospects of fuel cell technologies. Nat Sci Rev, 2017, 4: 163

5 Seh ZW, Kibsgaard J, Dickens CF, et al. Combining theory and experiment in electrocatalysis: Insights into materials design. Sci- 
ence, 2017, 355: 6321

6 Zhang J, Li Q, Wu H, et al. Nitrogen-self-doped carbon with a porous graphene-like structure as a highly efficient catalyst for oxygen reduction. J Mater Chem A, 2015, 3: 10851-10857

7 Liu X, Dai L. Carbon-based metal-free catalysts. Nat Rev Mater, 2016, 1: 16064-16076

8 Zhang G, Jin X, Li H, et al. N-doped crumpled graphene: bottomup synthesis and its superior oxygen reduction performance. Sci China Mater, 2016, 59: 337-347

9 Cao L, Yang M, Lu Z, et al. Exploring an effective oxygen reduction reaction catalyst via $4 \mathrm{e}^{-}$process based on waved-graphene. Sci China Mater, 2017, 60: 739-746

10 Li M, Zhang L, Xu Q, et al. N-doped graphene as catalysts for oxygen reduction and oxygen evolution reactions: Theoretical considerations. J Catal, 2014, 314: 66-72

11 Lee WJ, Lim J, Kim SO. Nitrogen dopants in carbon nanomaterials: defects or a new opportunity? Small Methods, 2017, 1: 1600014

12 Zhang J, Zhou H, Liu X, et al. Keratin-derived S/N co-doped graphene-like nanobubble and nanosheet hybrids for highly efficient oxygen reduction. J Mater Chem A, 2016, 4: 15870-15879

13 Zhang J, Zhou H, Zhu J, et al. Facile synthesis of defect-rich and S/ $\mathrm{N}$ co-doped graphene-like carbon nanosheets as an efficient electrocatalyst for primary and all-solid-state $\mathrm{Zn}$-air batteries. ACS Appl Mater Interfaces, 2017, 9: 24545-24554

14 Yang ZK, Lin L, Xu AW. 2D nanoporous $\mathrm{Fe}-\mathrm{N} / \mathrm{C}$ nanosheets as highly efficient non-platinum electrocatalysts for oxygen reduction reaction in Zn-air battery. Small, 2016, 12: 5710-5719

15 Tang C, Wang B, Wang HF, et al. Defect engineering toward atomic Co- $\mathrm{N}_{x}-\mathrm{C}$ in hierarchical graphene for rechargeable flexible solid Zn-air batteries. Adv Mater, 2017, 29: 1703185-1703192

16 Yan D, Guo L, Xie C, et al. N, P-dual doped carbon with trace Co and rich edge sites as highly efficient electrocatalyst for oxygen reduction reaction. Sci China Mater, 2018, 61: 679-685

17 Wu H, Jiang X, Ye Y, et al. Nitrogen-doped carbon nanotube encapsulating cobalt nanoparticles towards efficient oxygen reduction for zinc-air battery. J Energy Chem, 2017, 26: 1181-1186

18 Chen B, Bi H, Ma Q, et al. Preparation of graphene- $\mathrm{MoS}_{2}$ hybrid aerogels as multifunctional sorbents for water remediation. Sci China Mater, 2017, 60: 1102-1108

19 Sa YJ, Seo DJ, Woo J, et al. A general approach to preferential formation of active $\mathrm{Fe}-\mathrm{N}_{x}$ sites in $\mathrm{Fe}-\mathrm{N} / \mathrm{C}$ electrocatalysts for efficient oxygen reduction reaction. J Am Chem Soc, 2016, 138: 15046-15056

20 Artyushkova K, Kiefer B, Halevi B, et al. Density functional theory calculations of XPS binding energy shift for nitrogen-containing graphene-like structures. Chem Commun, 2013, 49: 2539

21 Yan D, Li Y, Huo J, et al. Defect chemistry of nonprecious-metal electrocatalysts for oxygen reactions. Adv Mater, 2017, 29: 1606459

22 Lee DU, Xu P, Cano ZP, et al. Recent progress and perspectives on bi-functional oxygen electrocatalysts for advanced rechargeable metal-air batteries. J Mater Chem A, 2016, 4: 7107-7134

23 Chung HT, Cullen DA, Higgins D, et al. Direct atomic-level insight into the active sites of a high-performance PGM-free ORR catalyst.
Science, 2017, 357: 479-484

24 Zhang J, Wu S, Chen X, et al. Egg derived nitrogen-self-doped carbon/carbon nanotube hybrids as noble-metal-free catalysts for oxygen reduction. J Power Sources, 2014, 271: 522-529

25 Guo D, Shibuya R, Akiba C, et al. Active sites of nitrogen-doped carbon materials for oxygen reduction reaction clarified using model catalysts. Science, 2016, 351: 361-365

26 Hang $\mathrm{C}$, Zhang J, Zhu J, et al. In situ exfoliating and generating active sites on graphene nanosheets strongly coupled with carbon fiber toward self-standing bifunctional cathode for rechargeable Zn-air batteries. Adv Energy Mater, 2018, 8: 1703539

27 Zhang L, Xia Z. Mechanisms of oxygen reduction reaction on nitrogen-doped graphene for fuel cells. J Phys Chem C, 2011, 115: 11170-11176

28 Zhang L, Niu J, Dai L, et al. Effect of microstructure of nitrogendoped graphene on oxygen reduction activity in fuel cells. Langmuir, 2012, 28: 7542-7550

29 Tang C, Wang HF, Chen X, et al. Topological defects in metal-free nanocarbon for oxygen electrocatalysis. Adv Mater, 2016, 28: 6845-6851

30 Imtiaz S, Zhang J, Zafar ZA, et al. Biomass-derived nanostructured porous carbons for lithium-sulfur batteries. Sci China Mater, 2016, 59: 389-407

31 Lee JS, Nam G, Sun J, et al. Composites of a prussian blue analogue and gelatin-derived nitrogen-doped carbon-supported porous spinel oxides as electrocatalysts for a Zn-air battery. Adv Energy Mater, 2016, 6: 1601052

32 Zhao H, Sun C, Jin Z, et al. Carbon for the oxygen reduction reaction: a defect mechanism. J Mater Chem A, 2015, 3: 1173611739

33 Daems N, Sheng X, Vankelecom IFJ, et al. Metal-free doped carbon materials as electrocatalysts for the oxygen reduction reaction. J Mater Chem A, 2014, 2: 4085-4110

34 Jeon IY, Zhang S, Zhang L, et al. Edge-selectively sulfurized graphene nanoplatelets as efficient metal-free electrocatalysts for oxygen reduction reaction: the electron spin effect. Adv Mater, 2013, 25: 6138-6145

35 Zhang C, Liu Y, Zhou J, et al. Tunability of photo-catalytic selectivity of B-doped anatase $\mathrm{TiO}_{2}$ microspheres in the visible light. Dyes Pigments, 2018, 156: 213-218

Acknowledgements This work was supported by the National Natural Science Foundation of China (51602113 and 51702111) and China Postdoctoral Science Foundation (2016M590692).

Author contributions Liu L designed and synthesized the samples; Zhang $\mathrm{J}$ and $\mathrm{Ma} \mathrm{W}$ characterized the samples and performed data analysis; Liu L wrote the paper with support from Huang Y. All authors contributed to the general discussion.

Conflict of interest The authors declare no conflict of interest.

Supplementary information Supporting data are available in the online version of the paper. 


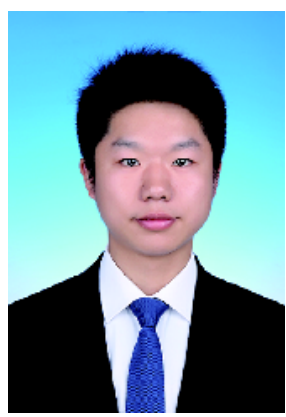

Lei Liu received his $\mathrm{PhD}$ in materials science from Wuhan University of Technology in 2016. Currently, he is a postdoctoral researcher at the School of Materials Science and Engineering, Huazhong University of Science and Technology. His research interests mainly focus on the design and synthesis of nanomaterials by element doping for energy storage and conversion applications.

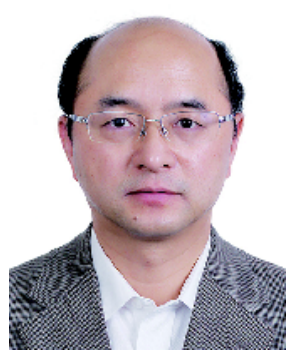

Yunhui Huang received his BSc, MSc and PhD degrees from Peking University. In 2000, he worked as a postdoctoral researcher in Peking University. From 2002 to 2004, he worked as an associate professor in Fudan University and a JSPS fellow at Tokyo Institute of Technology, Japan. He then worked in the University of Texas at Austin for more than three years. In 2008, he became a chair professor of materials science in Huazhong University of Science and Technology. His research group works on batteries of energy storage and conversion. For details please see the lab website: http://www. sysdoing.com.cn.

\section{钴/氮共掺杂多孔类石墨烯催化剂的制备及其高效氧还原性能表征}

刘磊, 张建 ${ }^{1,2}$, 麻伍军 ${ }^{3}$, , 黄辉 $^{1^{*}}$

摘要 本文通过纳米硅作为保护层结合高温热解得到一种新型钻/氮共掺杂多孔类石墨烯纳米碳材料氧还原催化剂(Co/N-GLC). 结果显 示, Co/N-GLC具有类似于石墨烯的碳纳米薄层结构, 并展现出分级多孔(微孔/介孔)特性, 其比表面积高达 $809 \mathrm{~m}^{2} \mathrm{~g}^{-1} ;$ 此外, Co/ $\mathrm{N}-\mathrm{GLC}$ 还 拥有较高的吡啶氮和石墨氮含量. 这些优异的特性使得 Co/N-GLC在碱性介质中具有出色的氧还原活性, 接近于商业 $\mathrm{Pt} / \mathrm{C}$ 催化剂. 同时, 在 锌空气电池测试中, Co/N-GLC具有接近Pt/C电极的放电性能和优异的稳定性, 表明该催化剂有望替代贵金属催化剂, 具有很好的应用前 景. 